\title{
Welcome to JEPS!
}

It is with great pleasure that we welcome readers to the inaugural issue of the Journal of Experimental Politics (JEPS). JEPS is the official journal of the Organized Section of the American Political Science Association (APSA) on Experimental Research. We are greatly honored to have been selected by the section as the first editors of the journal, and wanted to take this opportunity to briefly share with readers our vision for the journal, some interesting new-dare we say experimental? - policies that we have put in place for the journal, as well as the content in this first issue.

At its core, JEPS shares a common vision with the APSA Organized Section on Experimental Research: to provide a common space where scholars employing experimental methods in the study of politics and political behavior can share research and learn from one another about how to improve our research methodology. The section was originally established after a number of us realized that while there was an increasing amount of experimental research within the field of political science, most of the conversations were only taking place between people pursuing similar styles of experimental research: lab experiments (of both the game theoretical and social psychological varieties), field experiments, survey experiments, neurological experiments, etc. What was lacking, however, was a systematic opportunity for communication across these communities. This led the two of us to set up the first of the now annual NYU Center of Experimental Social Science (CESS) Conferences on Experimental Political Science. The interest in this conference - which was open to anyone using experiments to study politicswas one of a number of factors that helped provide a launching ground for the establishment of the APSA Organized Section, which subsequently established JEPS.

Accordingly, we take an expansive view of what it means to employ experimental methodology. A primary goal of the journal is to publish research in which investigators can randomly assign subjects to different treatment (and baseline) groups in an effort to be able to isolate and analyze causal effects, and the majority of the papers in this first issue are of that type (see Brancati, Bolsen et al., and Al-Ubaydli, et al.) However, we are also happy to consider work that is in that general spirit, such as so-called "natural" or "quasi-random" experiments or studies using "experimental reasoning" such as the article by Stadelman, et al. in this first 
issue. We are also interested in publishing work that furthers our understanding of experimental methods as in the article by Krupnikov and Levine, also in this issue. Similarly, we take a broad view of what it means that an article is focused on politics. If the topic or theoretical argument is likely to be of interest to political scientists, we are happy to consider it for publication in JEPS.

Our hope is that JEPS will become the journal of record for experimental research in political science, i.e., that eventually anyone who is using experiments in their research will feel it is important to publish some of that research in JEPS because that is where other scholars will look to see experimental research in political science (and, eventually, this is how the field develops). We want it to be a place where people who are interested in using experimental analysis in their own research will go to find ideas about how experiments can be employed, how to improve their own experimental research methodology, and, even for those not interested in using experiments in their own research, which is what we have learned from experimental analysis. Accordingly, we have established two criteria for review that may differ from those at other political science journals. First, we are explicitly instructing reviewers not to reject manuscripts because they do not have "a novel theoretical argument." We are perfectly happy to publish research with new theoretical arguments, but we are also just as happy to publish papers that use experiments in interesting ways to shed light on existing theoretical arguments. As an extension on that point, nor do authors need to be addressing a topic of interest to a "broad general audience." Again, if you are doing something of interest with an experiment in a research area that is relevant for political scientists, we are interested in considering your work for publication.

Second, we are happy to consider studies that replicate previous experiments. It is of course incumbent on the author to explain why a replication is interesting, but we believe that just because an experiment was run once utilizing a subject pool of University of Michigan undergraduates in the 1990s does not mean there is nothing new to learn from running the same experiment with a subject pool of Syrian armed combatants in 2014. More generally, we want to at least provide an opportunity for the accumulation of scientific knowledge that can occur when existing studies are replicated in new contexts.

Beyond publishing valuable content - which is of course our core goal—we are also trying to make the experience of reviewing for JEPS a pleasurable one, so much as any review experience can be pleasurable. We are well aware of the increasing demands being made upon members of our profession to volunteer unpaid time to review articles for journals. Accordingly, we have set up our entire organizational structure with the aim of making the review process work well for both authors and reviewers. The first step was bringing on board over a dozen top-notch Associate Editors (AEs) with a wide range of experimental specializations, one of whom is assigned to overview the review process of every submission. Having so many AEs allows us to make sure the paper gets into the hands of an editor who is familiar with the type of research in that particular manuscript. This procedure, in turn, 
means that the person inviting you to review a paper for JEPS is likely to be better able to judge whether that paper is in your research area, and thus the experience of reading the paper is likely to be more valuable and interesting to you.

Moreover, we are employing a number of strategies to reduce the burden on reviewers whenever possible. First, papers that are unlikely to have a chance of being published can be desk rejected without going out for review. This means that by the time you receive a request to review a paper for JEPS, someone with a degree of expertise in that area has already read the paper and decided that it at least has a chance of being of publishable quality. Second, our AEs have the authority to recommend acceptance of a revised paper without sending it back to reviewers if the AE feels the concerns of reviewers have clearly been satisfied; the AE can also elect to return the paper to only a limited number of reviewers in the second round of revision. Finally, we allow authors to submit reviews from previous journals as part of the review process at JEPS. Depending on our assessment of the prior reviews, this may allow us to assess the paper with fewer reviewers than for a new submission. Taken together, our goal is to reduce the frequency with which we need to draw upon our reviewer pool, as well as to ensure that when we do need to turn to reviewers, they are being asked to read papers that have already been screened at least once and are likely to be of interest to the reviewer. We know we will not succeed in all cases, but hopefully the organizational structure we have put in place will help further these goals.

We also hope that these policies will help make the process of submitting work for consideration for publication at JEPS a more pleasant one. The desk reject policy should help assure that papers that have no chance of being published in JEPS are quickly returned to their authors. The ability to submit prior reviews can lead to an expedited review process in which the author may receive a decision more quickly than usual. And again, our hope is that by employing a large number of AEs in an already methodologically specialized journal we can make it that much more likely that the review process surrounding any given submission is overseen by an editor with a degree of familiarity with the research subject, and that the ultimate reviews are that much more useful for authors.

But of course, the most important clientele for any journal is its readers, so we now turn to the task of introducing the content of the first issue of JEPS! We are presenting a cross-sample of modern political science experimental research, illustrating the maturation and diversity of experimentation as it is conducted in the discipline. That is, this issue contains examples of laboratory research, field and survey experiments, as well as research employing quasiexperimental reasoning. The versatility is also represented substantively; the first issue of JEPS includes work on political behavior and political economy, including topics such as electoral integrity (Brancati), collective action (Bolsen et al.), representation (Stadelmann et al.), and institutions (Al-Ubaydli, et al.). We also feature two articles related to experimental methodology, one on comparing differences in samples for experimental outcomes (Krupnikov and Levine) 
and another promoting reporting standards for experimental research (Gerber et al.).

The writings in this volume have in common an interest in the various aspects of information provision on individual and collective behavior. For example, Brancati presents a novel field experiment conducted during a naturally occurring election in Kosovo. She provides citizens with detailed information on how election monitors are involved in the election, by sending leaflets to households. Brancati's experiment points out the positive effects of information provision for citizens, yet these effects are not always guaranteed. The paper by Stadelman et al. also makes a noteworthy point in this manner. In their observational study, Stadelman et al. use a difference-in-differences strategy to identify the effect of an exogenous shock in the transparency of politician behavior on legislative actions taken. Surprisingly, Stadelmann et al. find that legislators do not change their behavior in a meaningful way, even though legislators become more observable. The contribution provided for this volume by Bolsen, et al. further provides a contrast to the articles by Brancati and Stadelmann et al., examining how citizens can be engaged into collective action. Their survey experiment addresses blame attribution from the perspective of collective action. Subjects are presented with frames that vary collective benefits and individual responsibility in the context of energy conservation. The treatment conditions cleverly exploit the variation in contents communicated to survey participants, allowing identification of the conditions under which collective action appears to be particularly feasible.

The last paper written by Al-Ubaydli et al. points out the importance of institutions. Their experiment uses incentivized controlled laboratory methods to test formally derived predictions on rent seeking behavior. Thus, in contrast to the methods employed in the previous article, the work of Al-Ubaydli et al. is set in a laboratory environment. The article addresses the emergence of the "resource curse" phenomenon. By manipulating the ability of subjects to communicate with and monitor each other, the authors show how institutions condition rent seeking. Moreover, the experimental design nicely connects traditional lab methods under a relatively high degree of control with a virtual world environment with realistic features in which subjects use avatars and interact, facing visually tangible tasks that can be easily manipulated by the researcher.

The final two contributions in the first volume address experimentation as a method. Krupnikov and Levine explore the difference between using different (student and adult general population) samples in a series of framing experiments. Their study shows sample differences largely in terms of effect size, but also between different modes of recruitment in the adult sample. The paper by Gerber et al. illustrates JEPS' role as the official journal of the Experimental Research Section of the APSA as it deals with reporting standards and guidelines for experimental research proposed by a committee of the Section. The guidelines should prove useful in ensuring "that scholars clearly describe what it is they did at each step in their research and clearly report what their data show." 
Once again, welcome to JEPS! It's been a long march from the first proposal that the APSA Organized Section on Experimental Research establish a journal to the publication of this inaugural issue, and there are way too many people to thank for making this happen. ${ }^{1}$ But everyone should know that a lot of hard work from many people went in to bringing this day to fruition, and we hope you enjoy the results!

\author{
Rebecca B. Morton \\ Joshua A. Tucker \\ New York University
}

\footnotetext{
${ }^{1}$ We must, however, recognize our graduate student assistants at NYU for special thanks. Sönke Ehret and Marko Klašnja have been working with us since the journal launched last summer, and we are very pleased that Emine Deniz has recently joined us as well. The graduate assistants have been instrumental in many facets of the journal's development, including the preparation of this introductory piece.
} 